\title{
High Precision Timing Measurement in the CALICE Analogue Hadronic Calorimeter.
}

\section{NSS-2016}

Motivation

2 The CALICE AHCAL engineering prototype

SPS CERN Campaign

$\geqslant$ Timing Calibration

2 Results and Conclusion

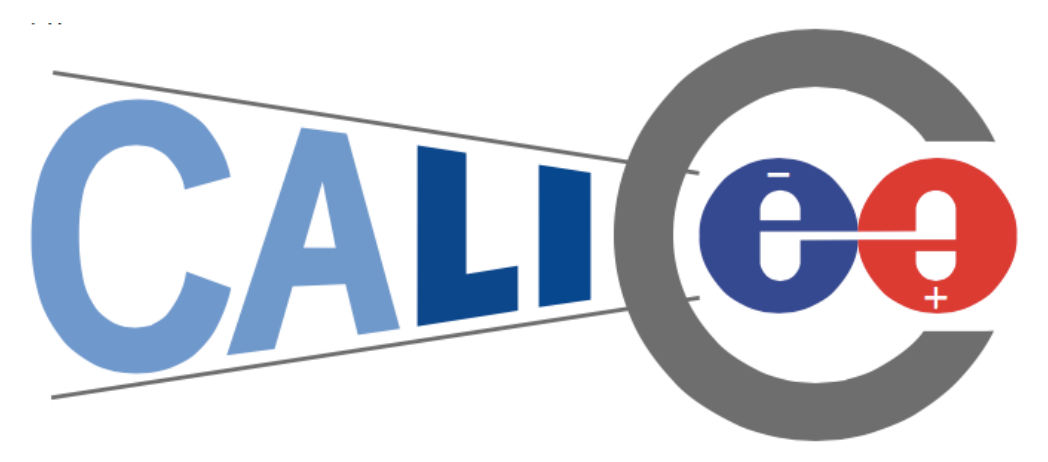

Eldwan Brianne for the CALICE

Collaboration

IEEE Strasbourg 2016

$03 / 11 / 16$ 


\section{Motivation for precision timing}

Timing may help:

- Resolving components in hadronic showers -> ns

- Separation of vertices -> 20-30 ps $\sim 1 \mathrm{~cm}, 1 \mathrm{~ns} \sim 30 \mathrm{~cm}$

- Timing based clustering/Energy reconstruction -> ns

- Background rejection and pile-up mitigation -> ns

ILC beam structure

- $\sim 300$ ns bunch spacing $->\sigma_{t}<300$ ns

- No need to disentangle bunch crossings

CLIC beam structure

- $\sim 0.5 \mathrm{~ns}$ bunch spacing $->\sigma_{t} \ll 0.5 \mathrm{~ns}$

- Timing is very important here to associate particles to correct bunch crossing 


\section{The CALICE AHCAL}

Highly granular Hadronic calorimeter concept readout with Silicon Photo-multipliers (SiPMs)

Active material: plastic scintillator

Designed for a future $\mathrm{e}^{+} \mathrm{e}^{-}$linear collider (ILC)

Technological prototype: Scalability to a collider detector and mass production

- Front-end integration/performance (see M. Reinecke poster - N24-2)

- DAQ (see J. Kvasnicka talk - N63-1)

Contributions to time resolution:

- SiPM 10 - 100 ps rise time (dependant of number of p.e)

- Scintillator $\sim$ ps rising time

- Light propagation to the photodetector $\sim$ ps to ns

- Electronics -> ps to ns

\section{Scheme for ILC}

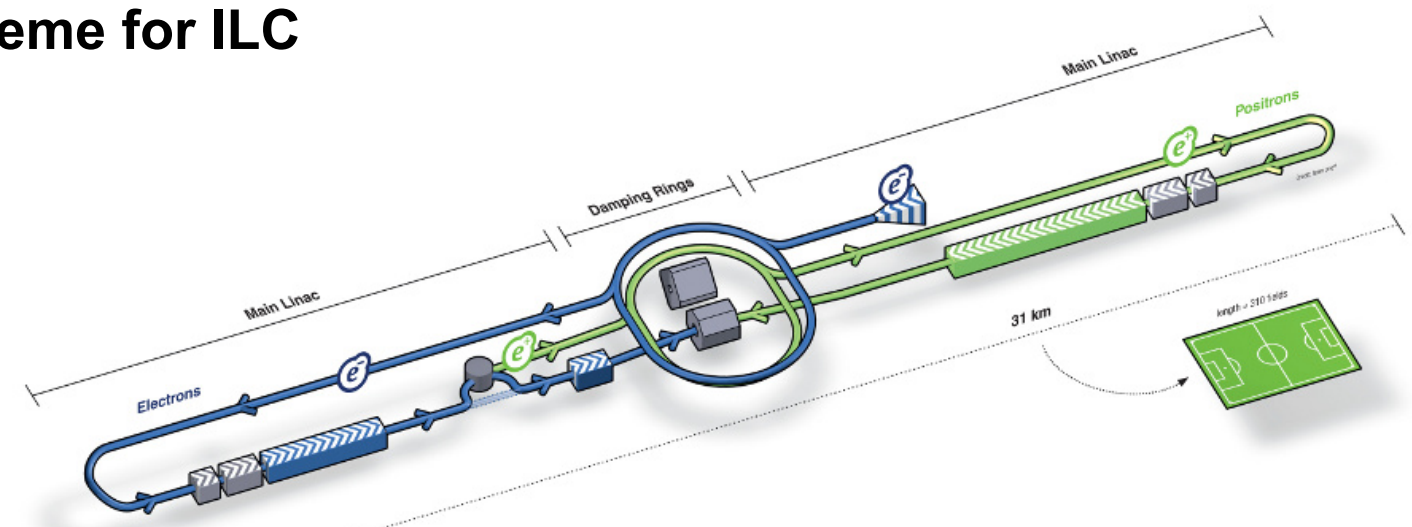

HCAL Board Unit

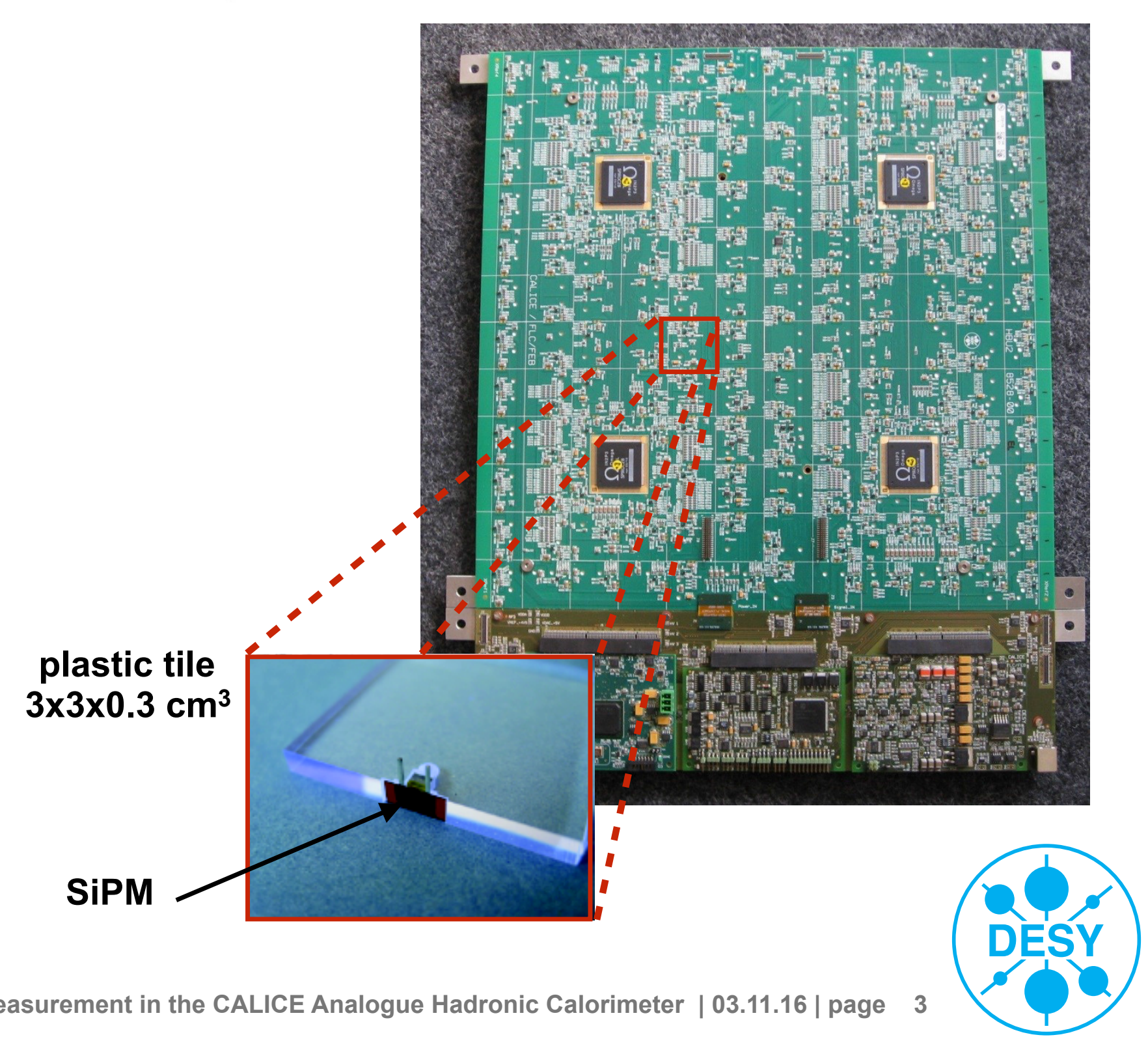




\section{Testbeam at CERN SPS}

Testbeam campaign at CERN in July 2015

Goals:

- Test of different tile/SiPM designs

- Check EM performance of the detector

- Study of timing of hadronic showers in 3D (radial, longitudinal)

Setup:

- 14 layers ( 3800 channels)

- Trigger signal $\left(T_{0}\right)$ directly fed to the chip as a normal channel $\rightarrow$ reference time

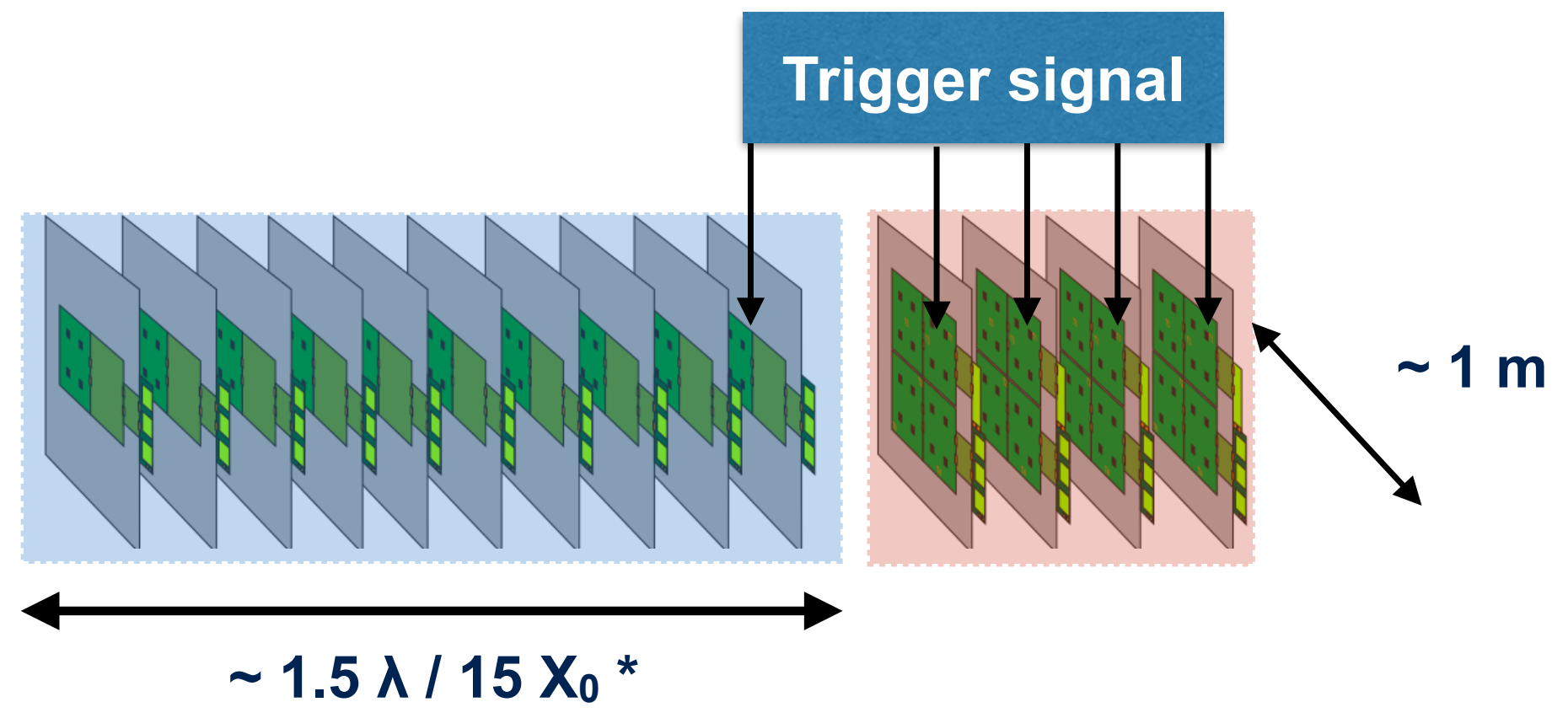

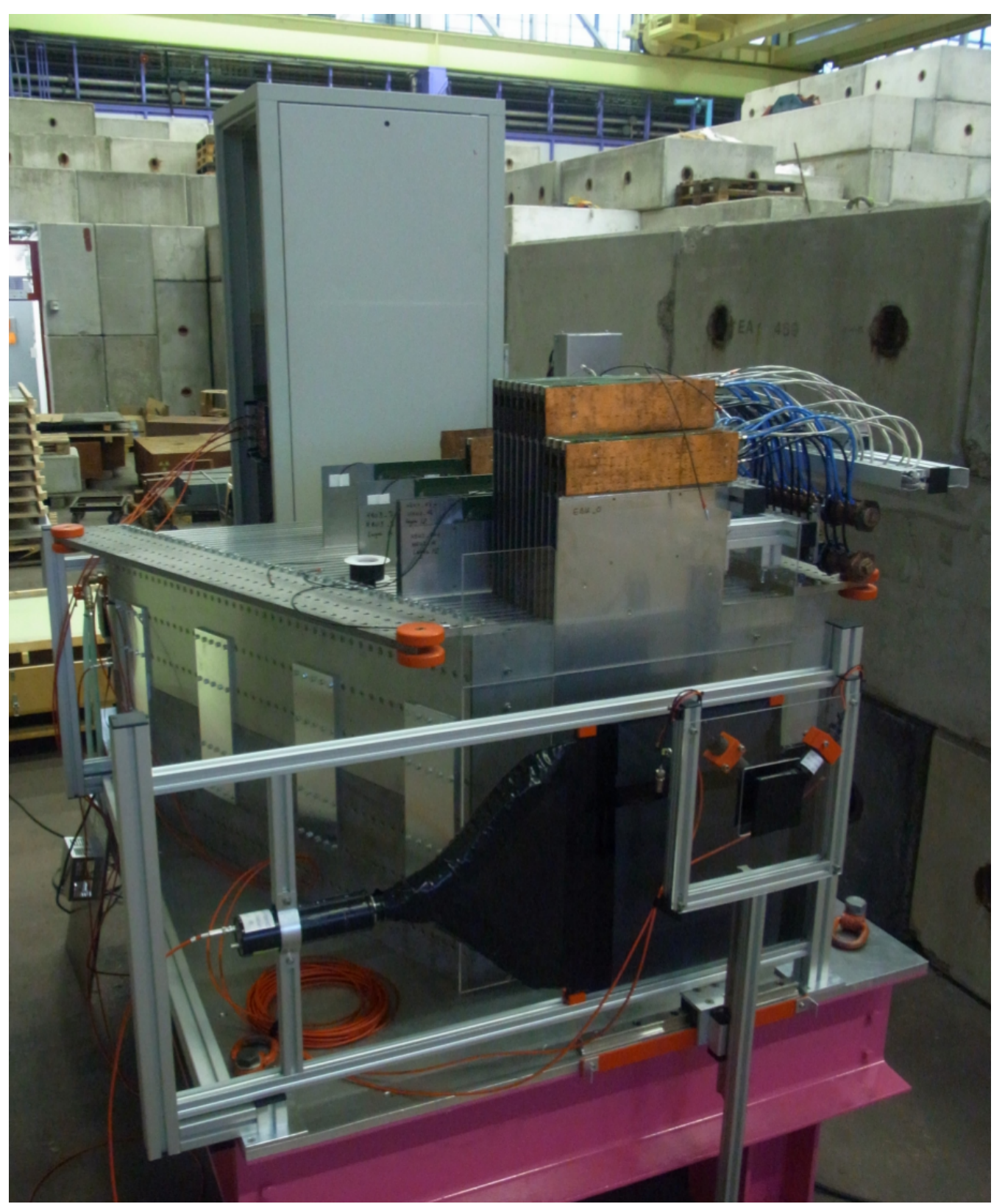

Picture of AHCAL in steel stack @ CERN 


\section{The readout chip : SPIROC2b}

Self-triggered chip / 36 channels

Only the first hit is registered in a clock period

Energy measurement :

- Analog measurement / 12 bits

- 16 memory cells per channel

Time measurement :

- ADC sampling of 2 voltage ramps

- 16 memory cells per channel

- Testbeam (250 kHz BX) : $\rightarrow \sim 1.6 \mathrm{~ns}$ per bin

- Design to run synchronous to the ILC machine $(5 \mathrm{MHz} \mathrm{BX})$-> time resolution $\sim$ x20 better

Calibration of analogue components is needed.

\section{clock \\ single ramp \\ ramp 1 \\ ramp 2 \\ combined}
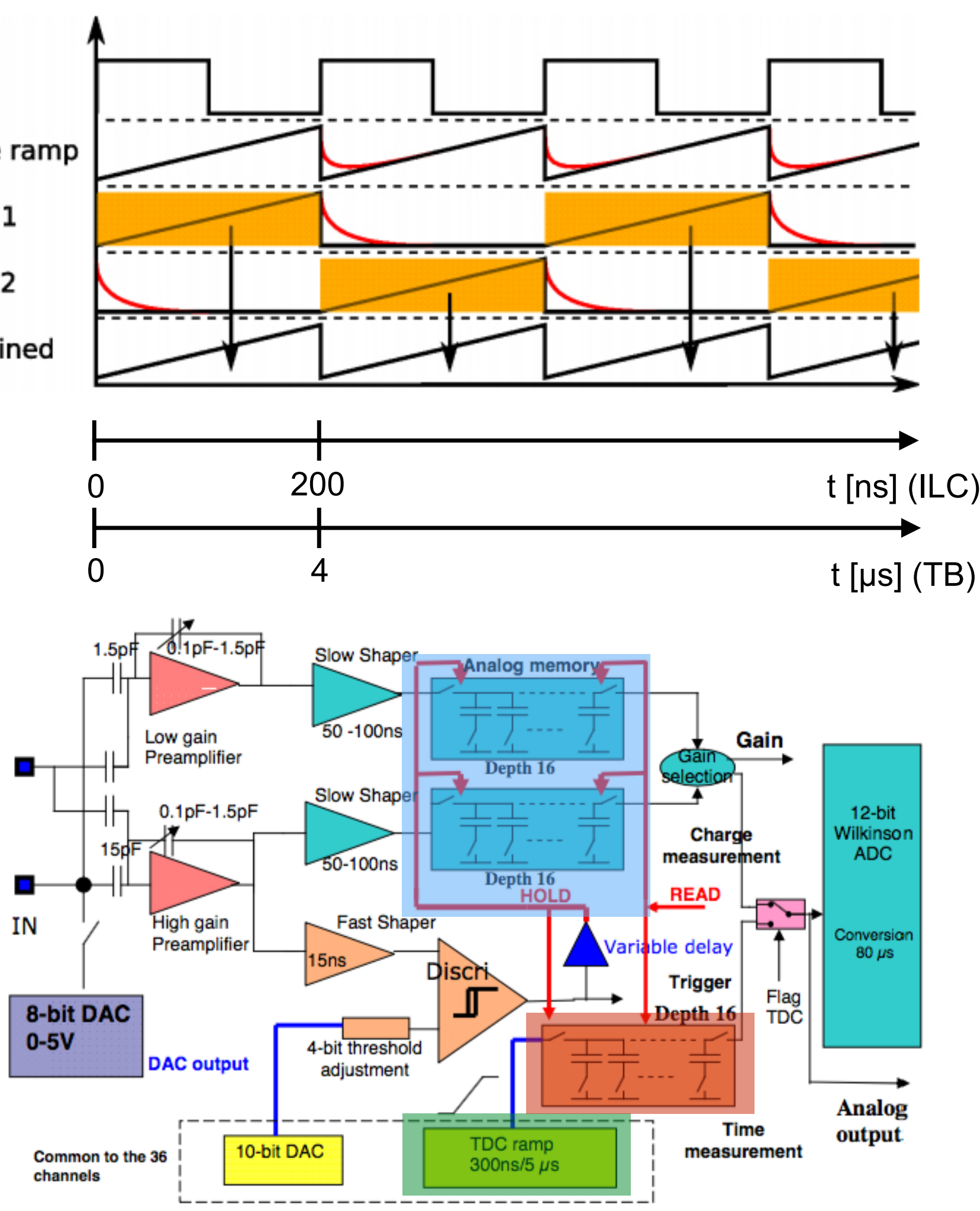


\section{Conversion from TDC to nanoseconds}

Timing is measured via a voltage ramp and stored in a memory cell

- Assumed to be linear

- Not possible to measure directly the ramp (too many chips) -> developing a robust procedure to extract the slope of the ramp

Need to use instantaneous particles -> muons for calibration, electrons for validation

\section{Procedure:}

- Measuring starting point and end point of the ramp on the TDC Spectrum

- Total ramp length in TB $(250 \mathrm{kHz}) \sim 4000 \mathrm{~ns}$ (3920 ns due to dead-time)

- Extract slope for each chip, BXID

- Extract pedestal for each chip, channel and first memory cell

In the order of expected value: $\sim 1.6 \mathrm{~ns} /$ TDC

slope $_{c h i p, B X I D}[\mathrm{~ns} / \mathrm{TDC}]=\frac{3920 \mathrm{~ns}}{\operatorname{Max}_{c h i p, B X I D}-\text { Pedestal }_{c h i p, B X I D}}$

$\mathrm{t}[\mathrm{ns}]=\left(\mathrm{TDC}-\right.$ Pedestal $\left._{\text {chip,chn,Mem=1 }}\right) \times \operatorname{slope}_{\text {chip }, B X I D}$
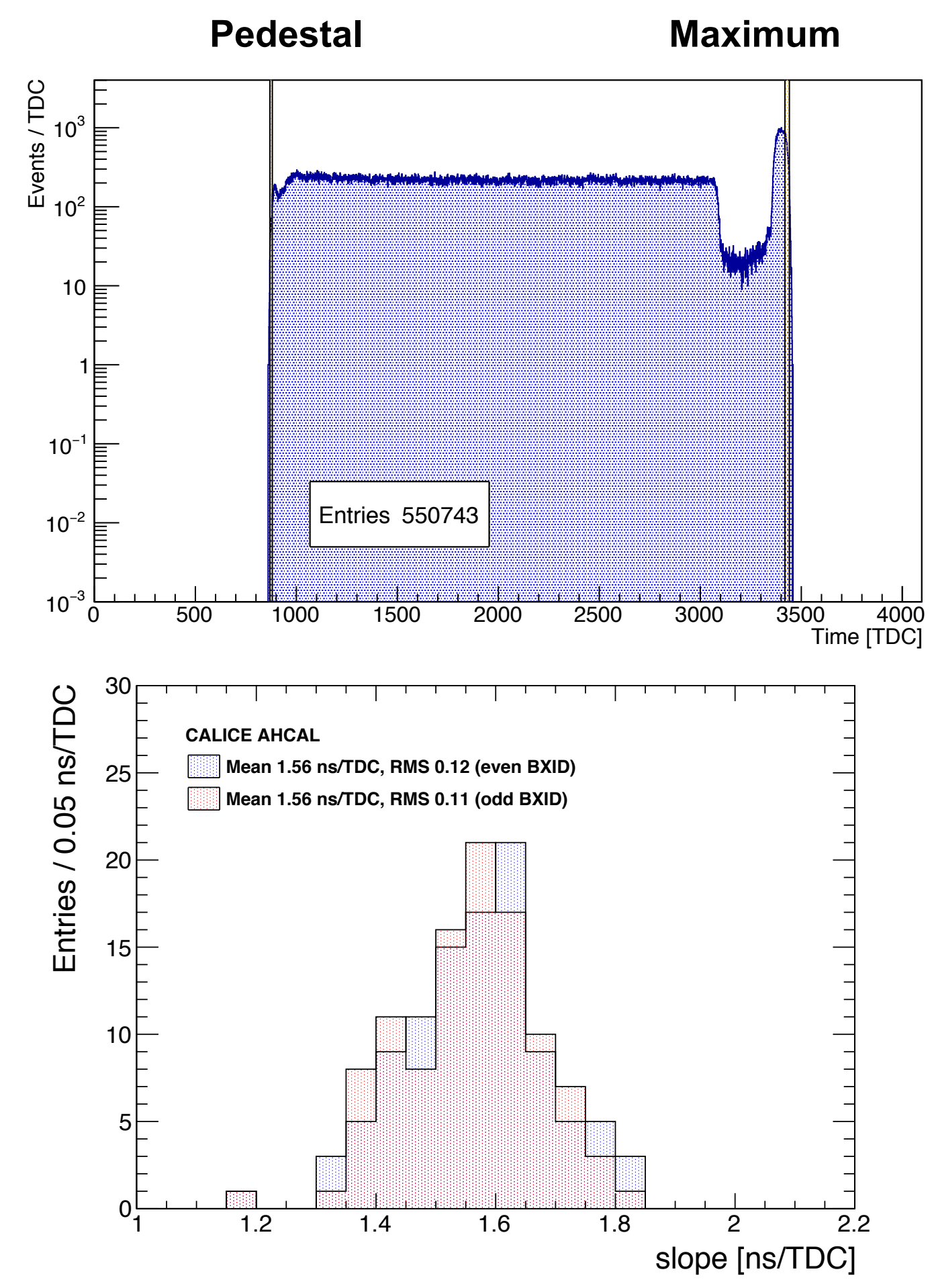


\section{Calibration of the time reference}

Time reference correction per ramp type (BXID odd/even)

The time of the trigger is needed in order to have the relative time of a particle $->$ need proper calibration

4 channels in the AHCAL receiving the trigger signal from scintillators

The trigger signal is coming from the exact same source

- Time of the trigger should be the same between channels.

- Additional offset needed due to different pedestal values per ramp BXID for each memory cells

Trigger resolution $\sim 4 \mathrm{~ns}$

Electronics contribution in final resolution
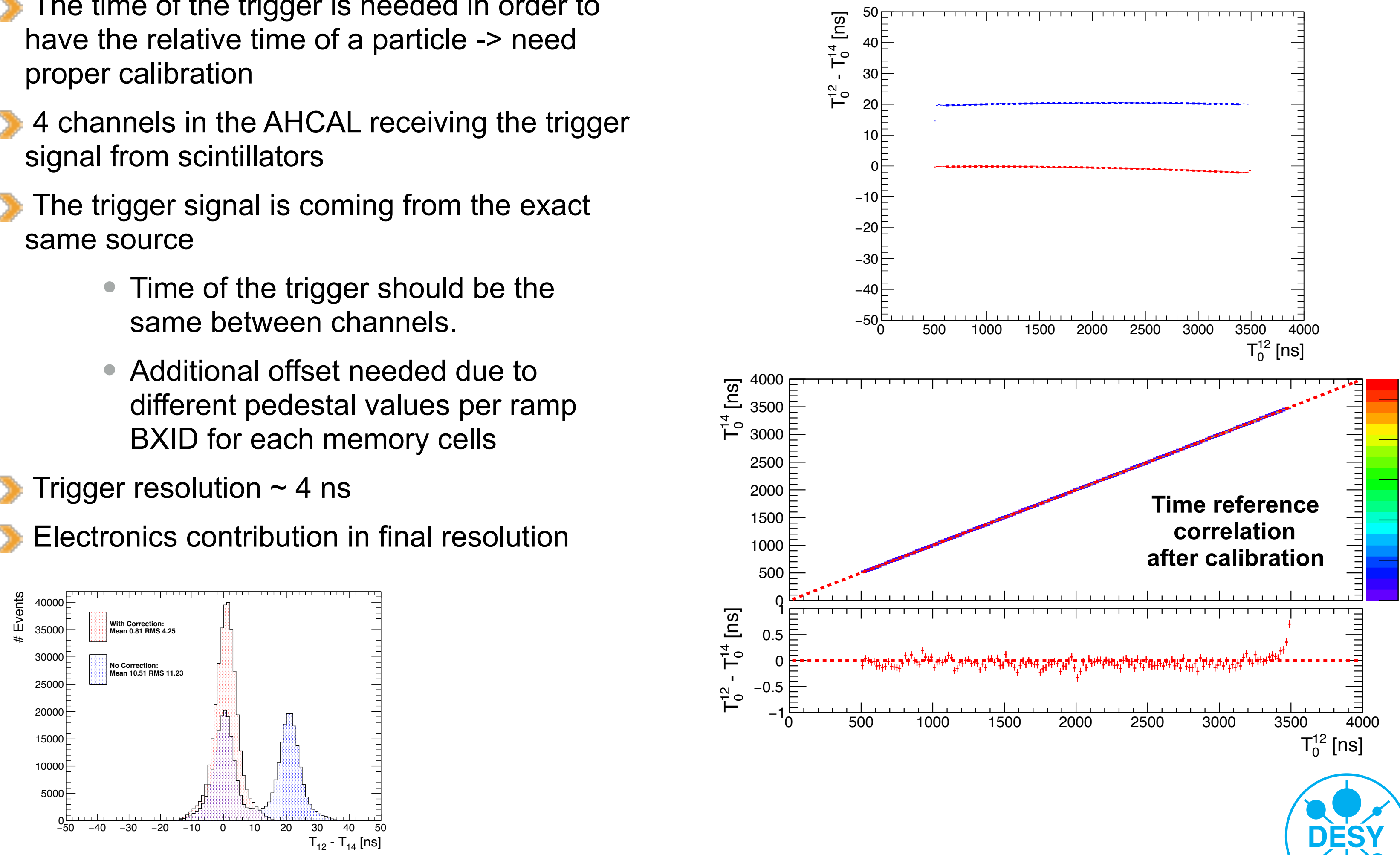

Eldwan Brianne | High Precision Timing Measurement in the CALICE Analogue Hadronic Calorimeter | 03.11.16 | page 


\section{Time of first hit}

Previous calibration applied similarly to all channels

Time of first hit is determined by looking at the time of the hit relative to the time of the trigger

- Slightly asymmetric distribution

- Time resolution 5.7 ns RMS

Possible improvements due to effects known from the electronics

- Non-linearity of the voltage ramp

- Time-walk effect due to the threshold

- Pedestal shift with high number of hits in a chip
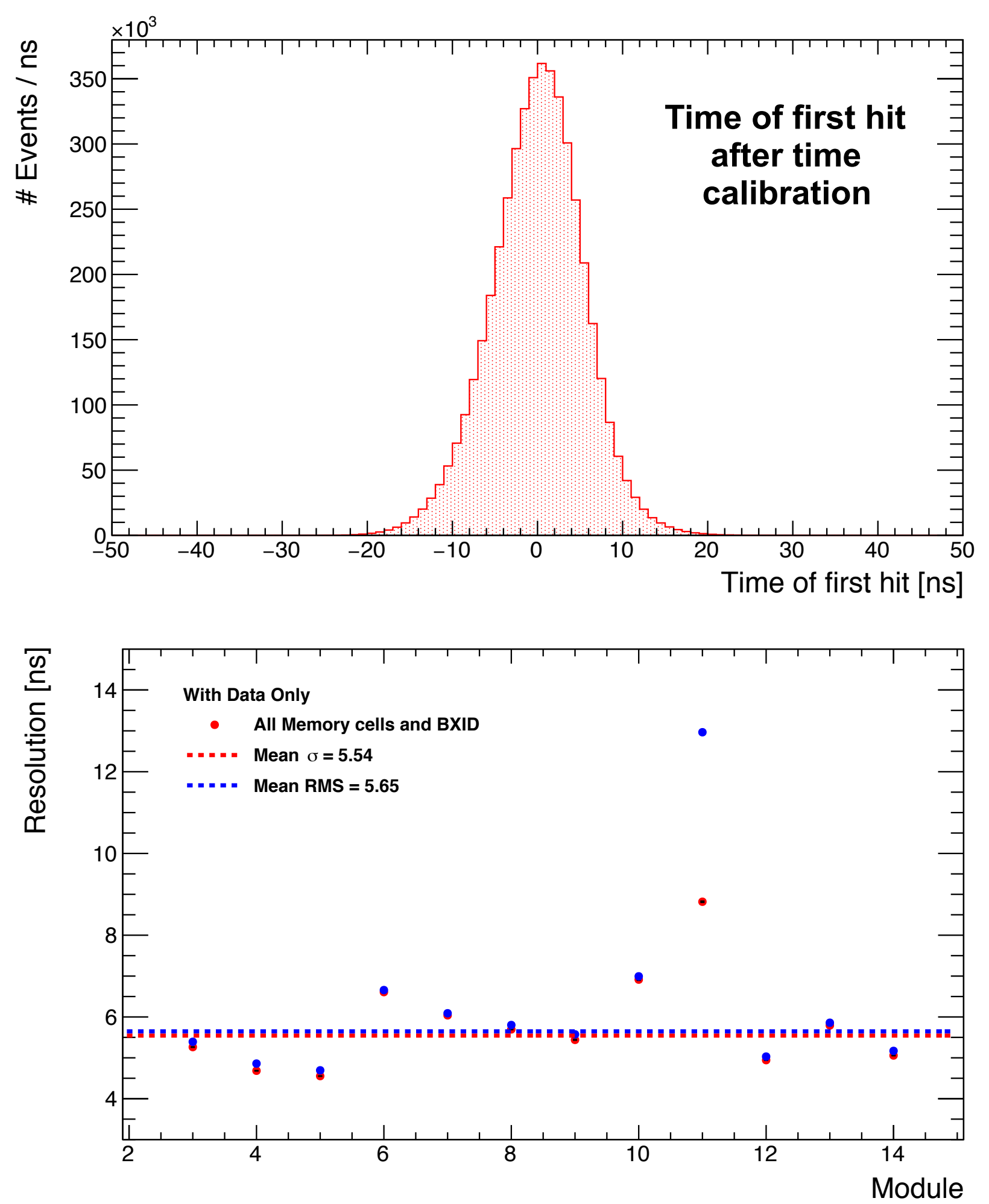


\section{Ramp non-linearity correction}

Assumption of a linear voltage ramp would mean:

- Time of the hit versus the TDC value of hit would yield a flat distribution -> no dependency of where the hit occurred in the ramp

2 Dependency observed

Correction with a 2nd order polynomial done for each chip and ramp BXID (odd/ even)

Improvement in the order of $6 \%$
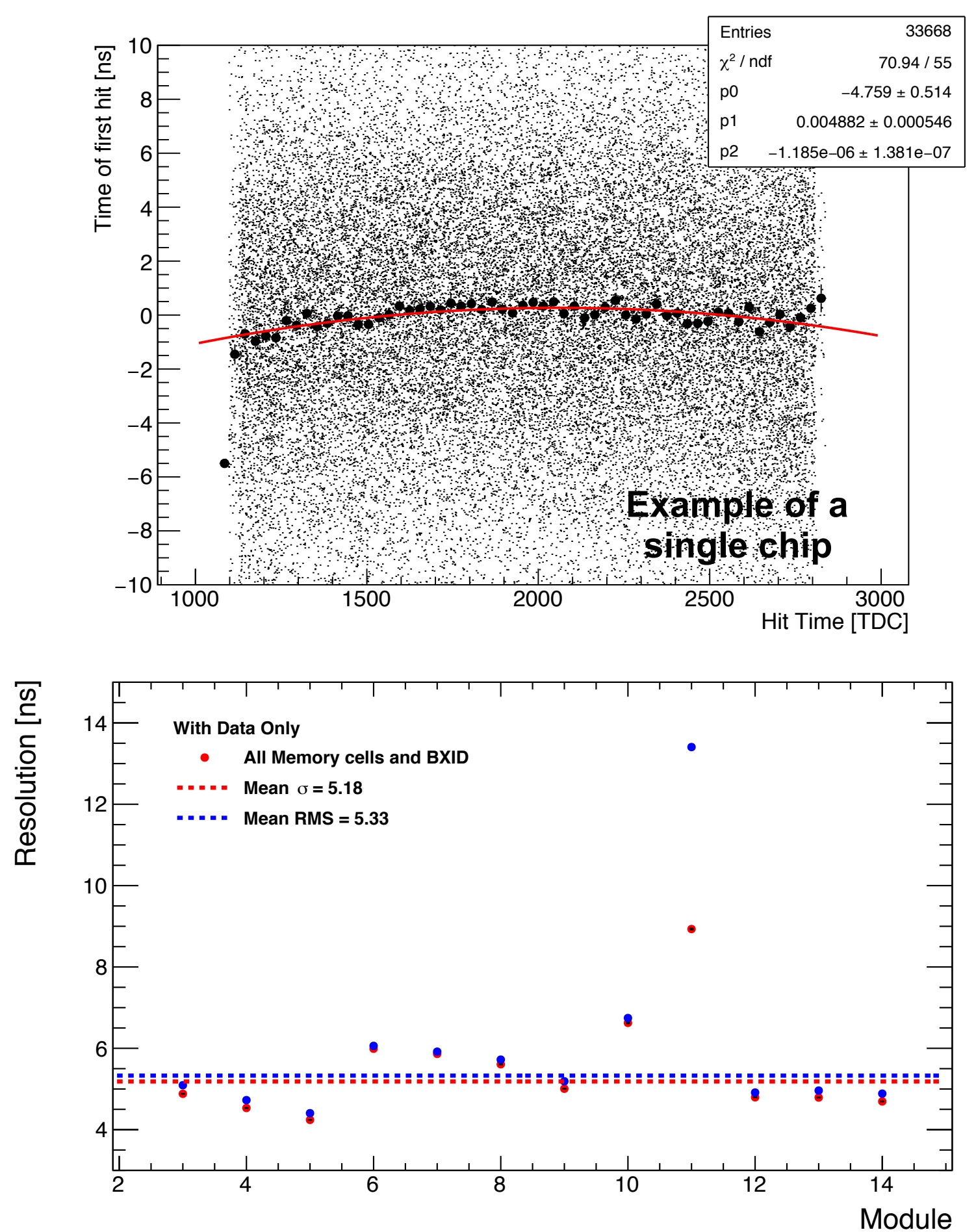


\section{Time-Walk correction}

Threshold -> dependency with hit energy

- Low amplitude hits triggers later than high amplitude hits

Fit with a exponential function and assumed to be global

Corrections up to $\sim 6$ ns between low energy hits and high energy hits

\section{Improvement in the order of $4 \%$}

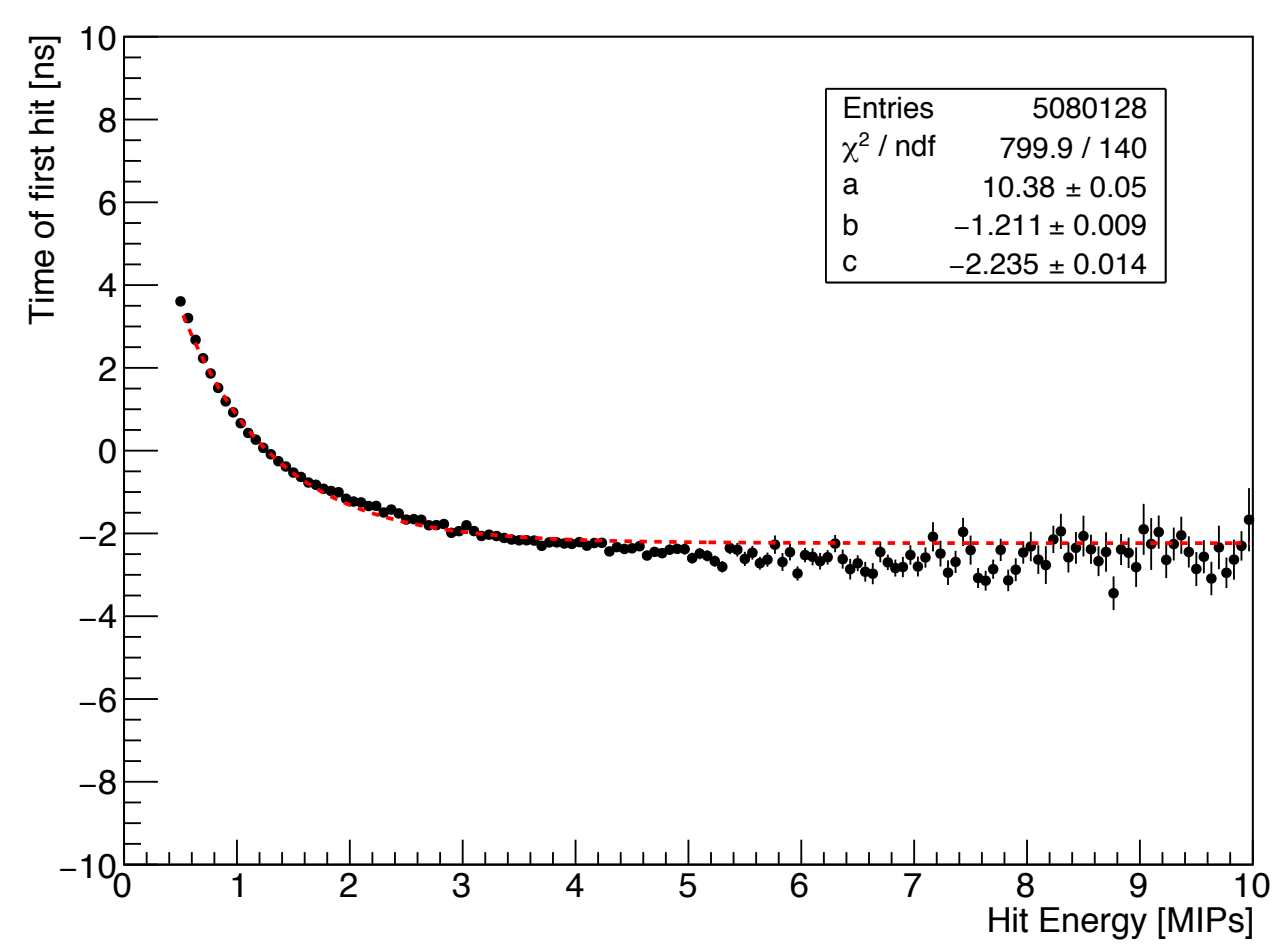

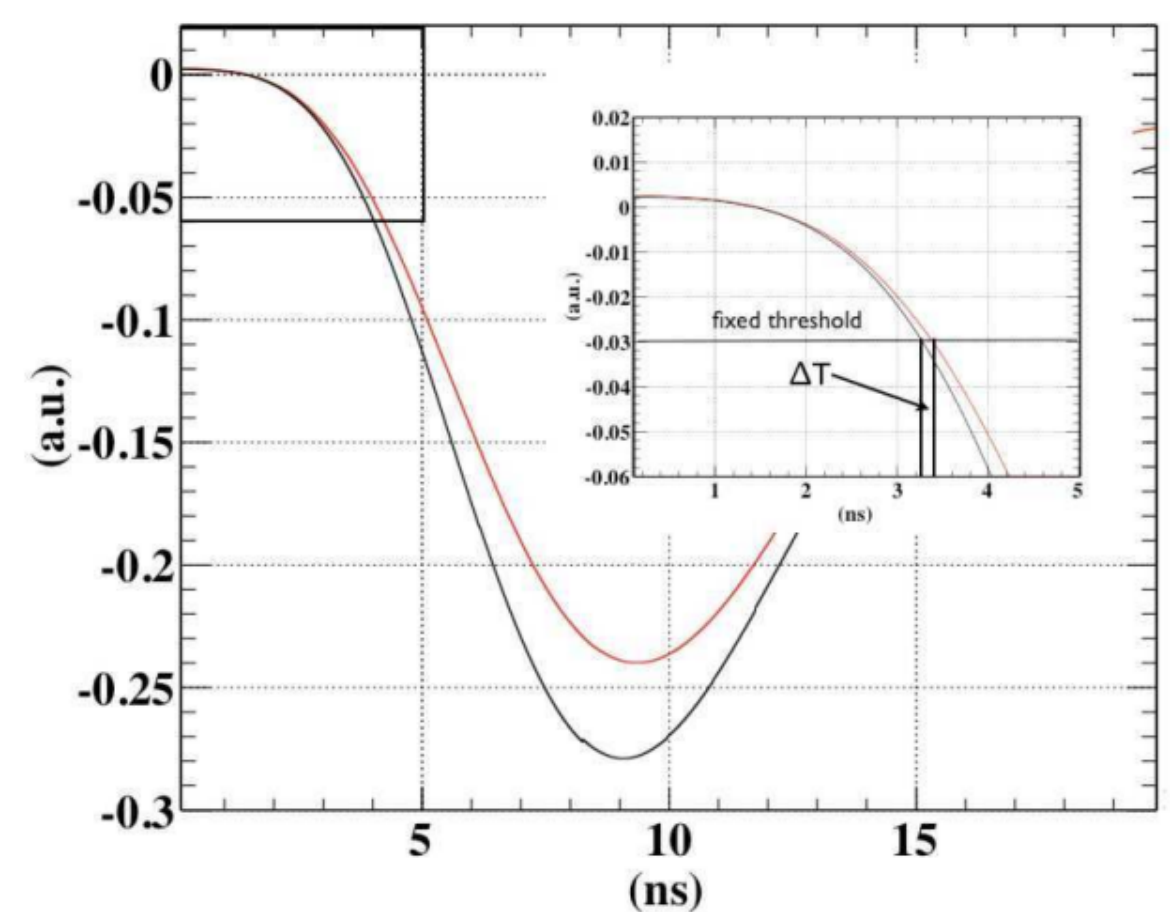

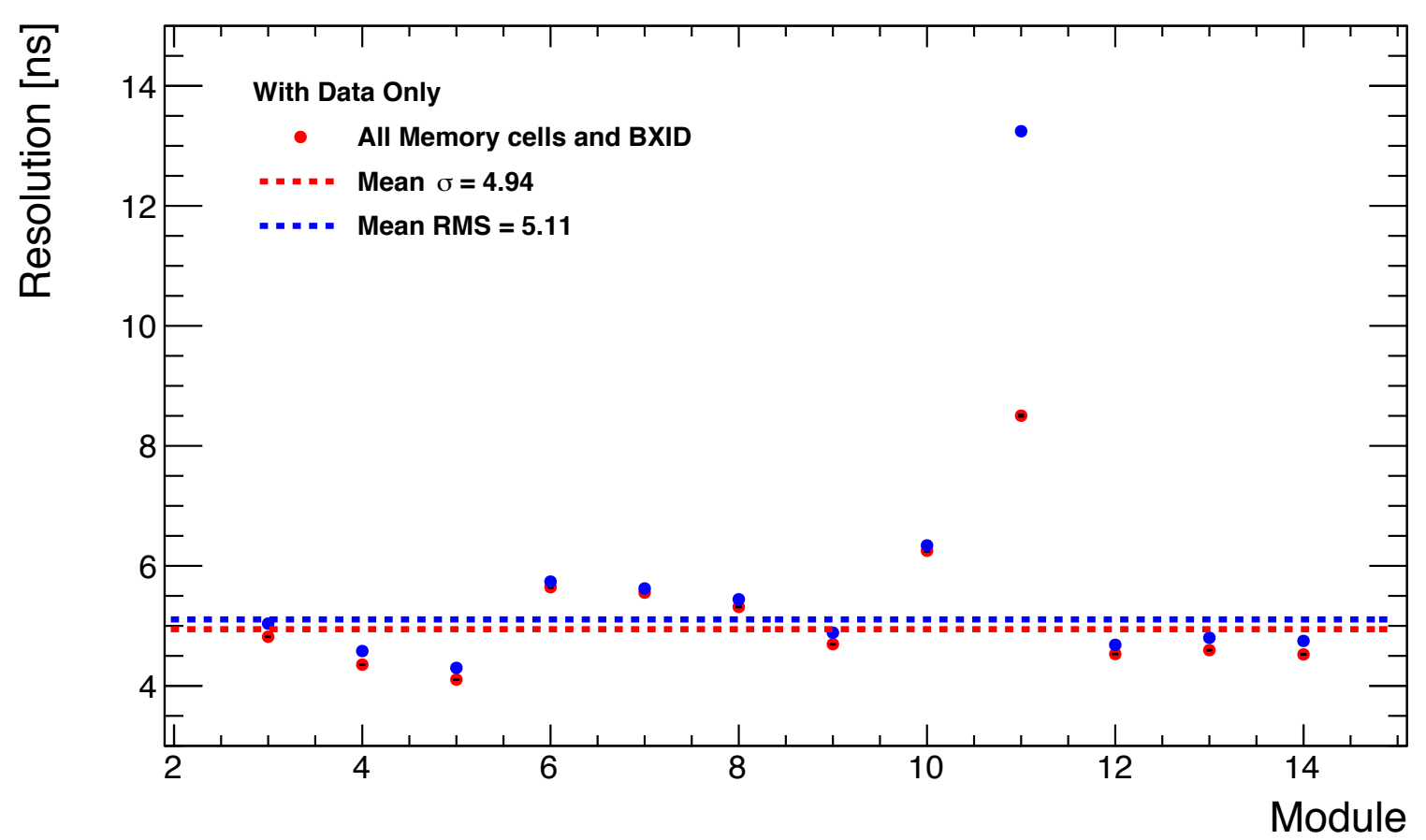




\section{Number of hits correction (electrons)}

Expected offset compared to muons (different trigger setup) $\sim 13 \mathrm{~ns}$

When several channels of a single chip are saturated -> shift in the ADC pedestal position can be observed

- Electronics effect $->$ too much current flowing trough the chip leading to a decrease of the power supply of the pre-amplifier

Similar effect for the TDC is observed -> increase of the time of hit with the number of hits in a chip

Increase of the time resolution up to 10-15 ns for large number of hits -> relevant for electrons and hadron showers
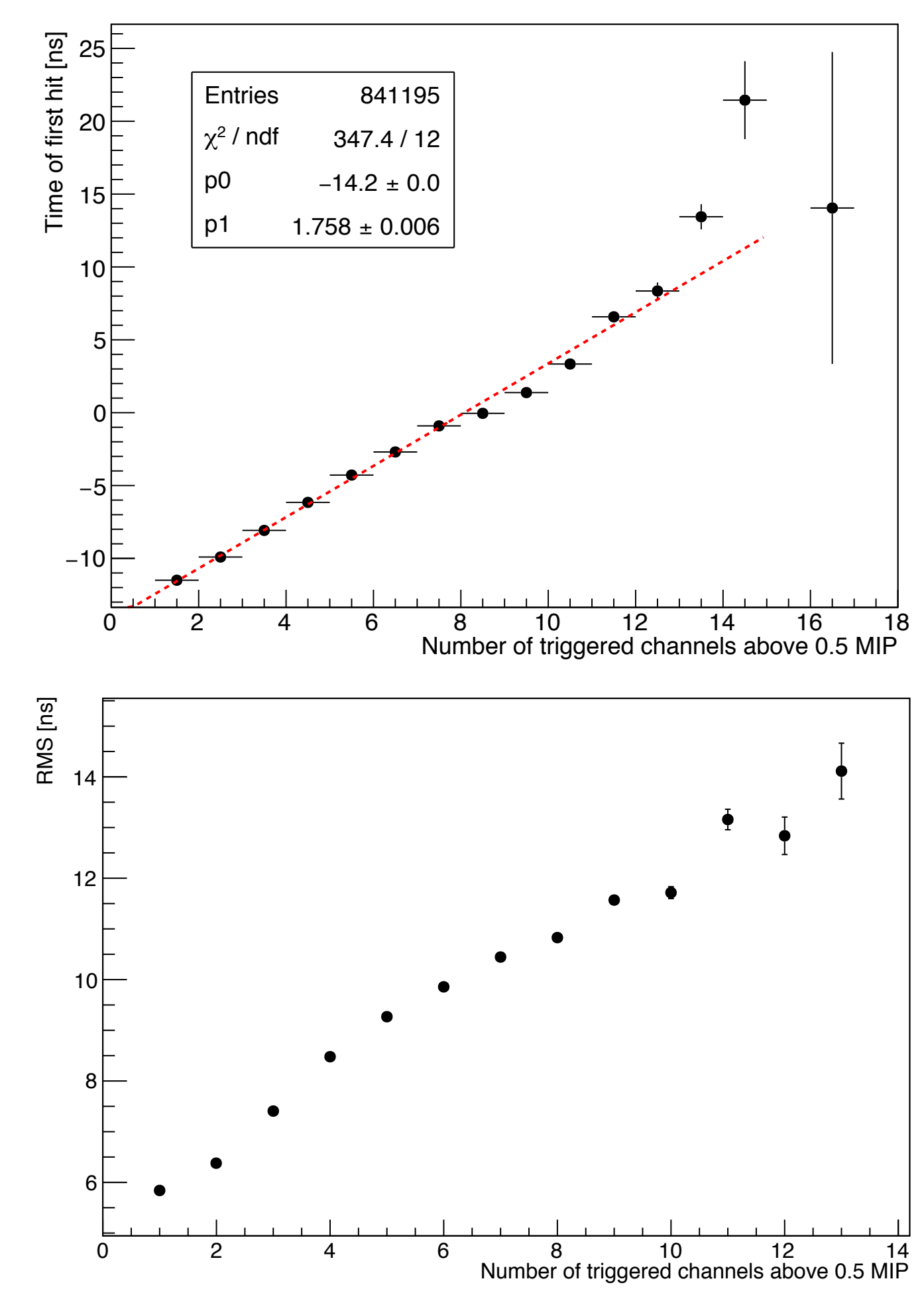


\section{Results}

\section{Muons}

- Resolution 5 ns RMS

- Fit with double gauss function -> take into account asymmetry

- Use parameters extracted from data for simulation

- $\sim 20 \%$ max deviation over $[-20,20]$ ns range

\section{Electrons}

- Resolution 7.8 ns RMS

- Single hit distribution -> very similar to muons

- MC ongoing -> introduction of number of hits effect
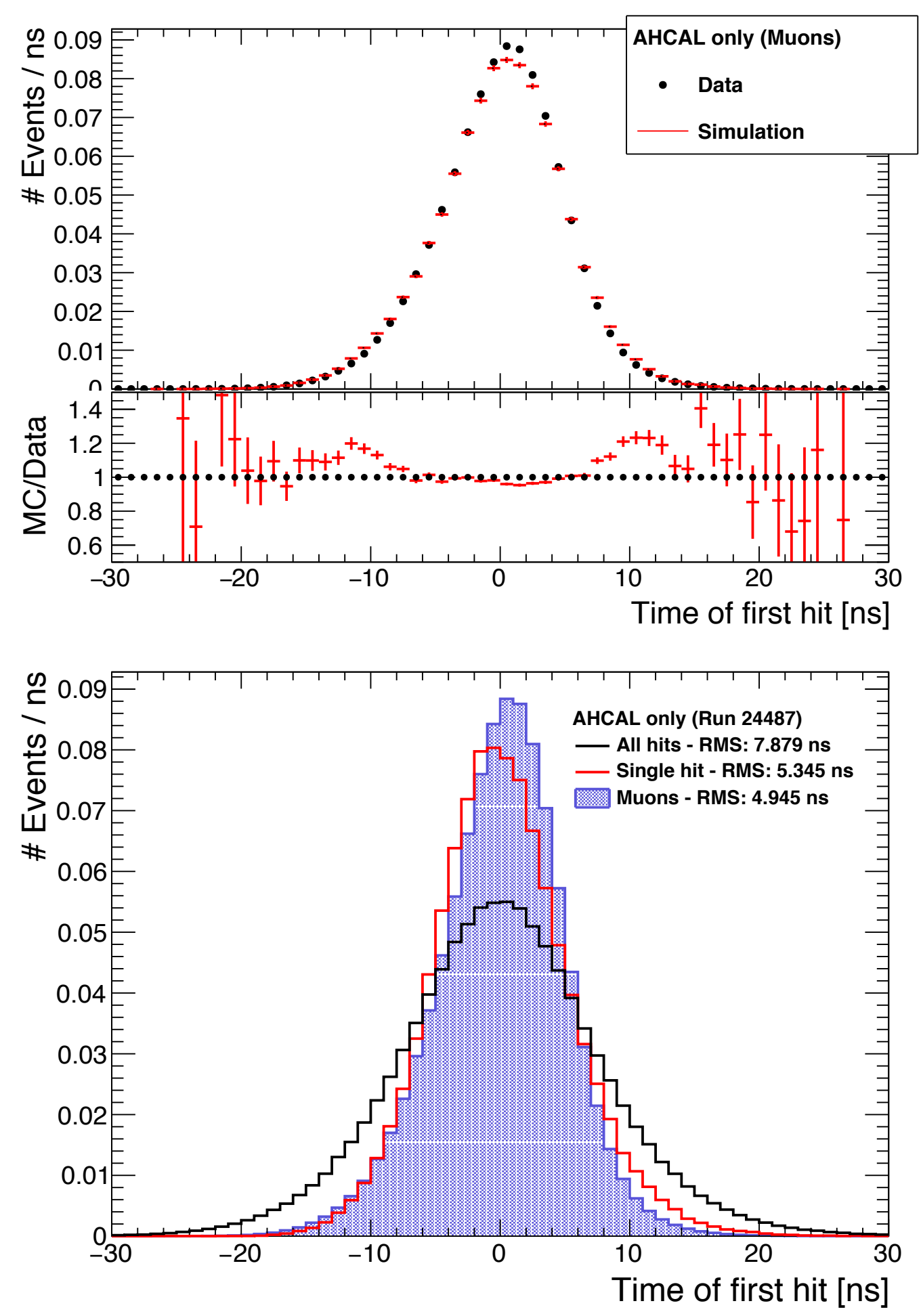


\section{Conclusion and Outlook}

A procedure to calibrate timing in the CALICE AHCAL has been developed

Timing resolution in testbeam with SPIROC2b achieved between 5 to $7.8 \mathrm{~ns}$-> should be enough to study timing in hadronic showers

Extrapolation to ILC (clock period $5 \mathrm{MHz}$ ) -> $400 \mathrm{ps}$ if electronics are the dominating effect in time resolution (if influence of scintillator and SiPM negligible)

Outlook:

- Look into pion data: time dependance in 3D (radial and longitudinal evolution)

- Short/long term correlations between layers for hadronic showers

- Study of impact of the use of timing in background rejection, pattern recognition and energy reconstruction

New chip (Spiroc2e) with improved pedestal shift and TDC scheme -> should improve the time resolution 


\section{Backup}

Eldwan Brianne | High Precision Timing Measurement in the CALICE Analogue Hadronic Calorimeter | 03.11.16 | page 14 


\section{The International Linear Collider}

Future e+e- linear collider

- $32 \mathrm{~km}$ length

- Energy : 250 - $500 \mathrm{GeV}$ (upgrade to $1 \mathrm{TeV}$ possible)

- Luminosity :

Planned to be build in Japan

2 detector concepts (ILD and SiD)

2 Physics goals:

- Precise Higgs mass / top mass measurements

- Higgs self-coupling

- BSM

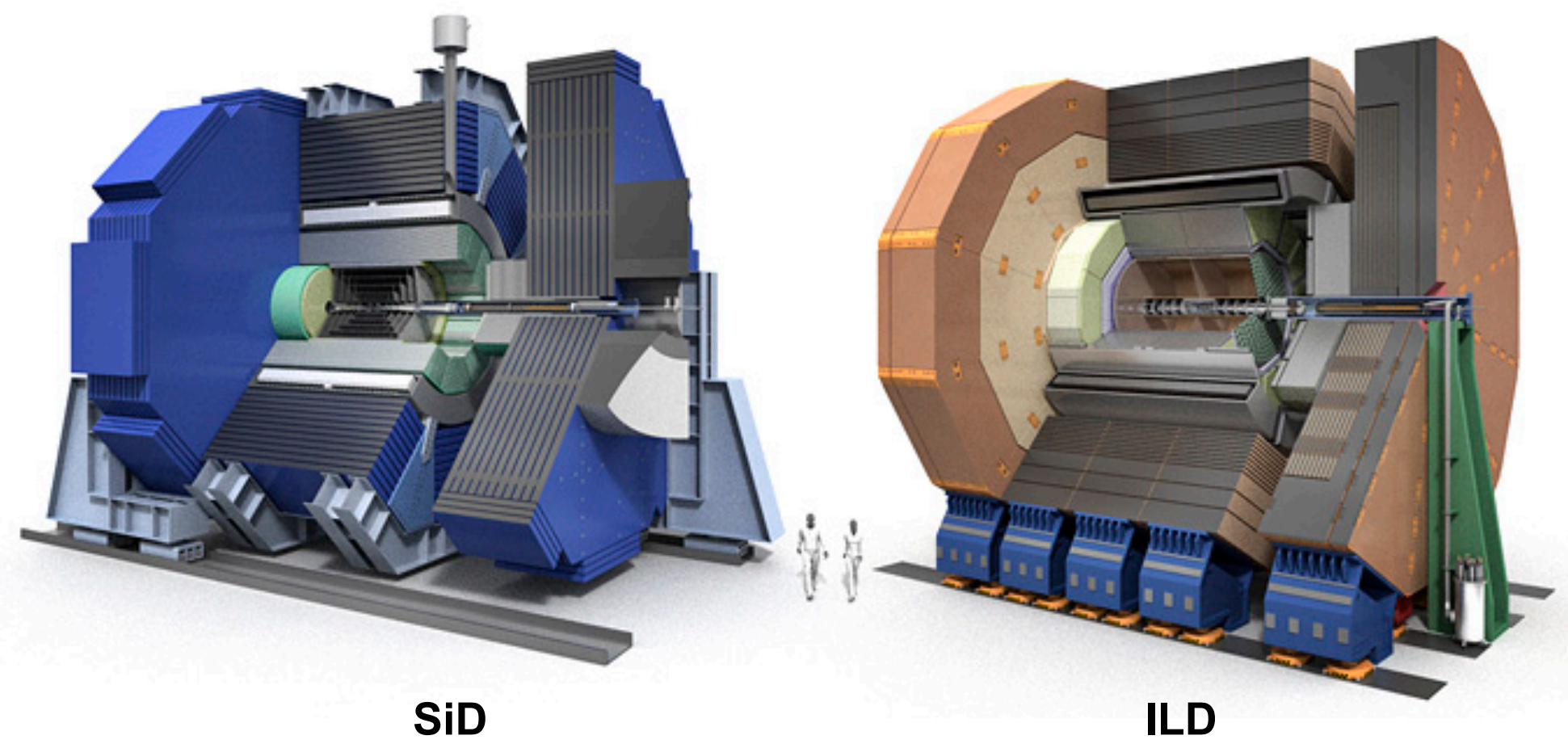




\section{SIPM used in CALICE AHCAL Technological prototype}

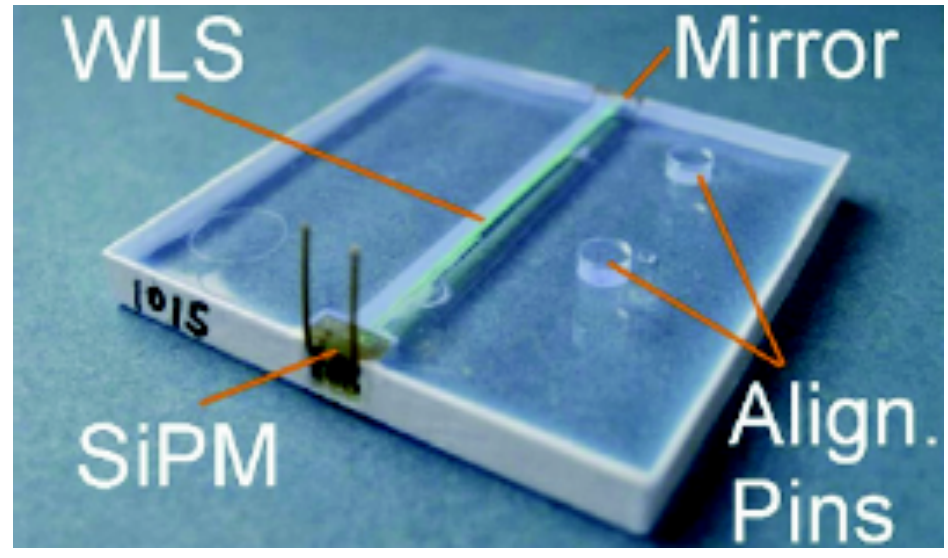

Old ITEP tiles with WLS fiber $800 \mathrm{px}$

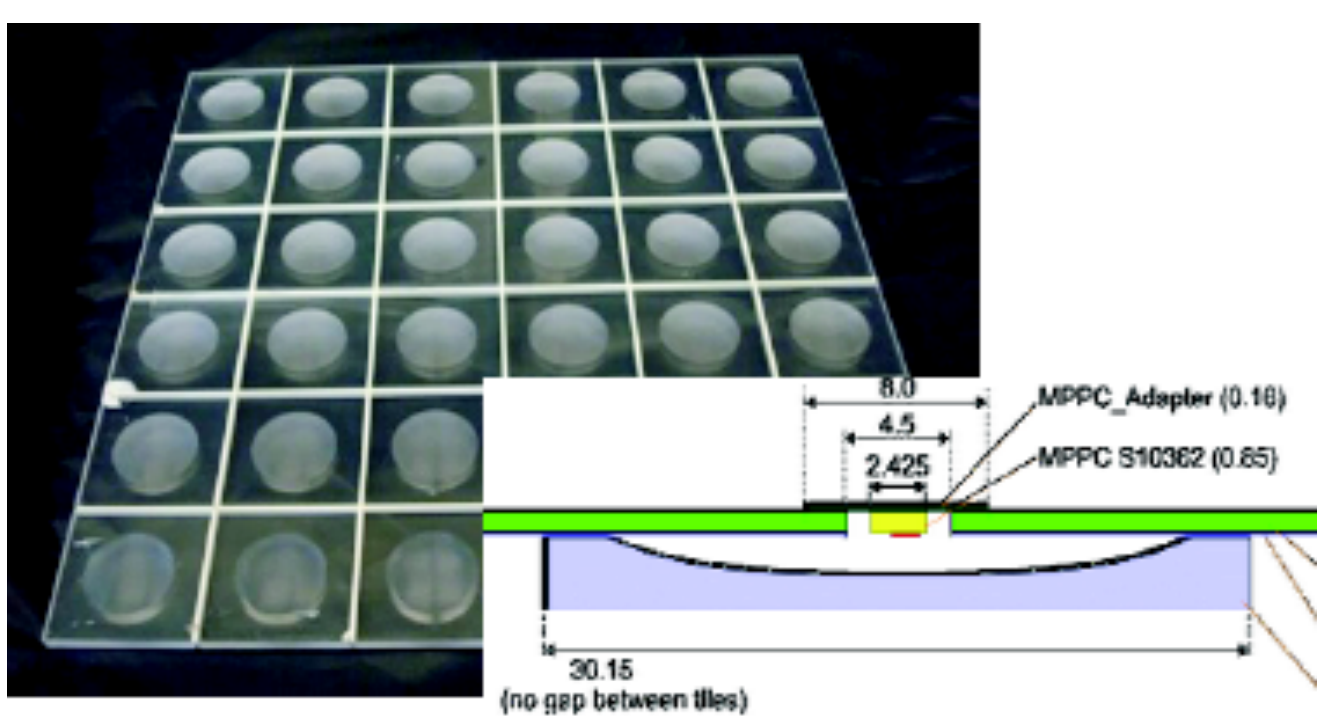

NIU megatile SMD MPPC 1600 px (50 $\mu \mathrm{m})$ MPPC $25 \mu \mathrm{m}$

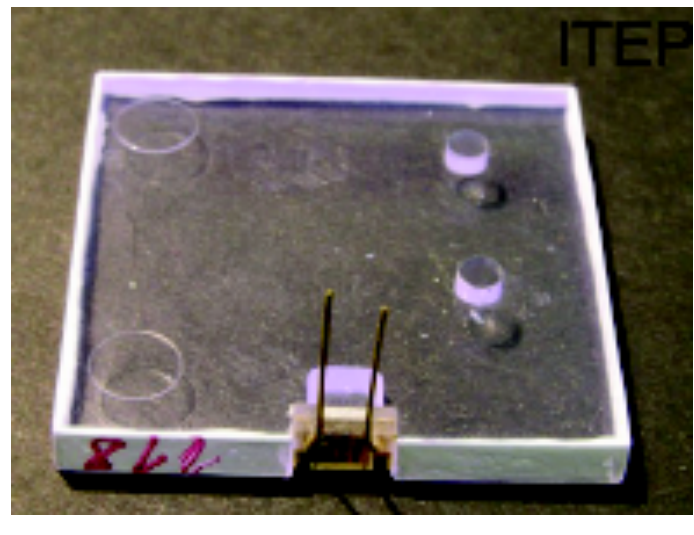

New ITEP tiles Ketek 12k px

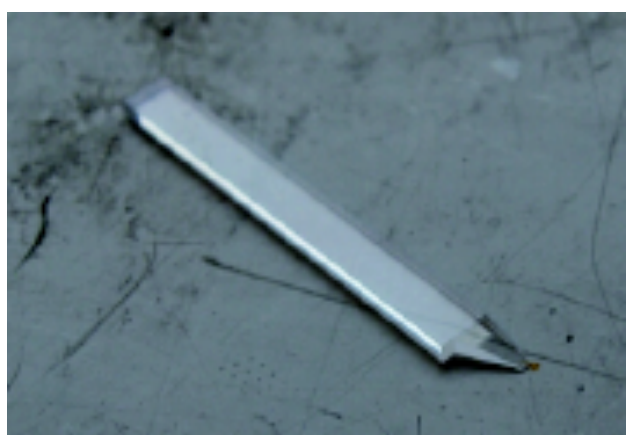

EBU strip Hamamatsu MPPC 10k px - 3600 px

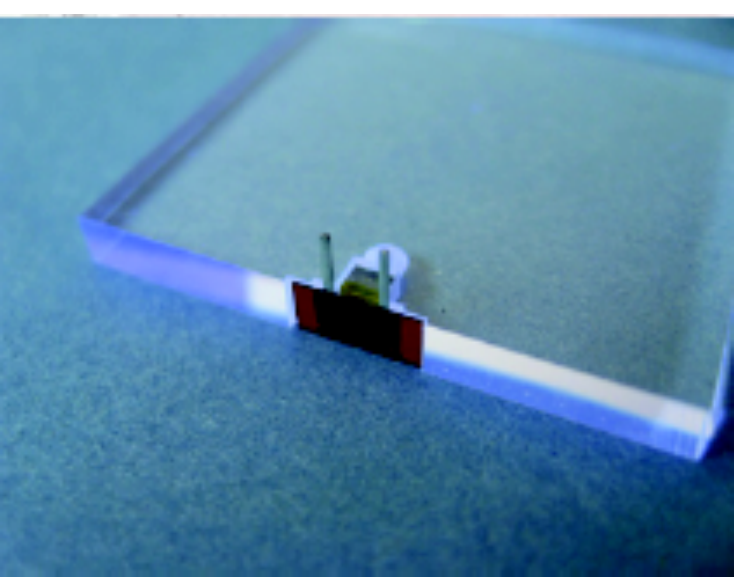

UHH + Heidelberg wrapped tiles

Ketek 2300 px SenSL 1300 px 


\section{Layer 11 - Electronics problem}

Layer 11 shows significant worse time resolution

- Due to noisy TDC ramp -> double ramp present for all chips

- Difficulties to extract the ramp slope

- Worsen the time resolution significantly

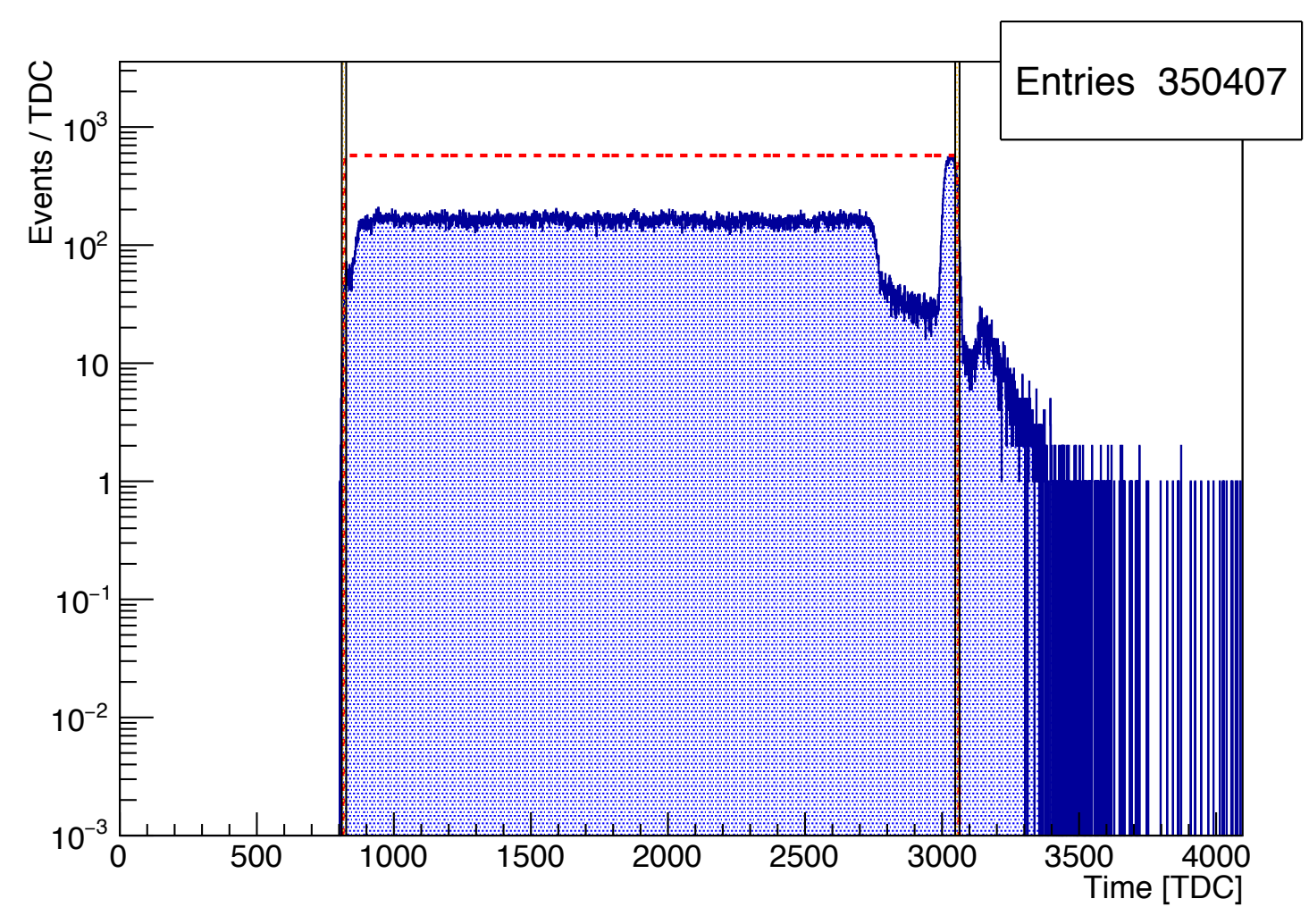

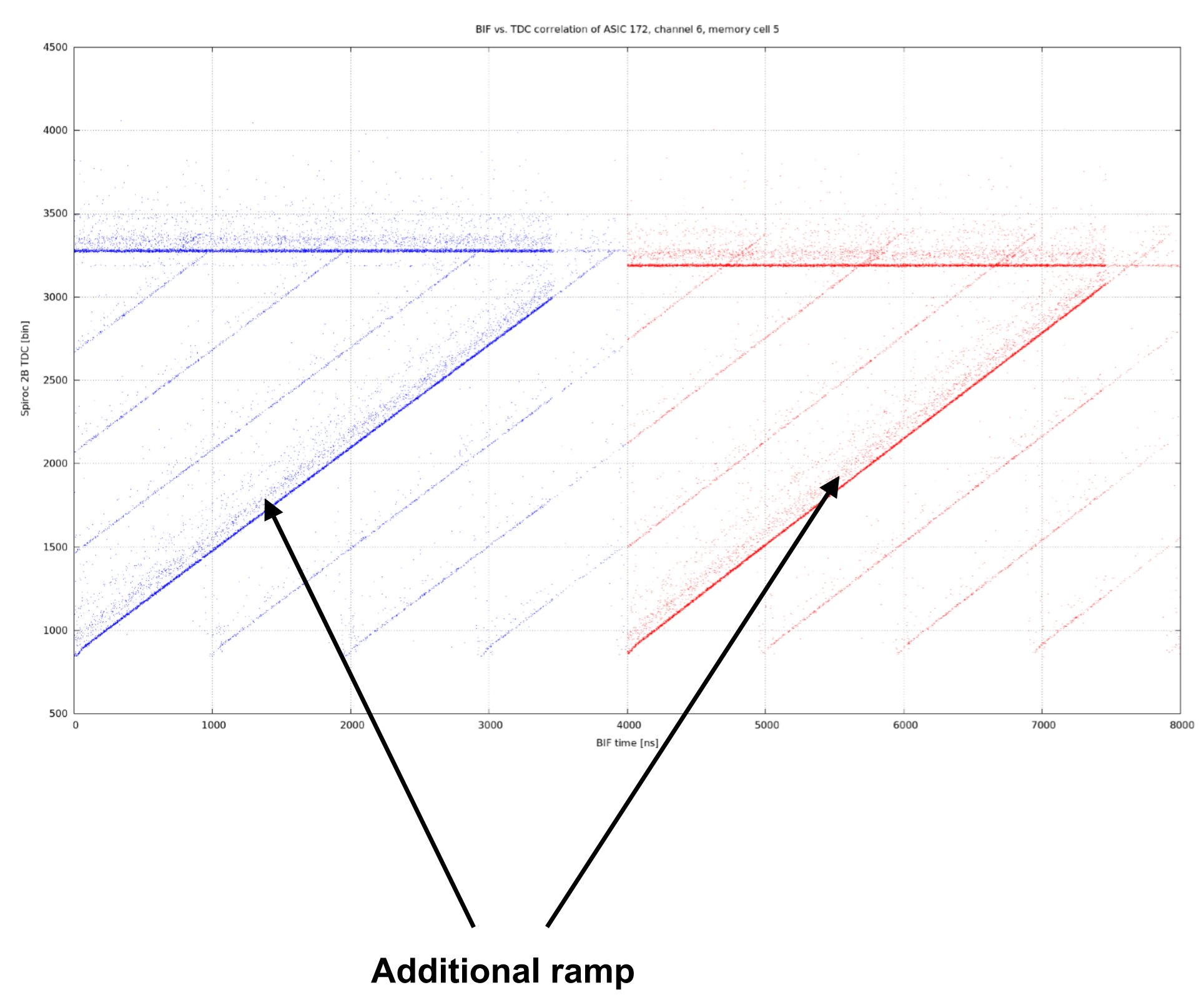




\section{SPIROC Acquisition}

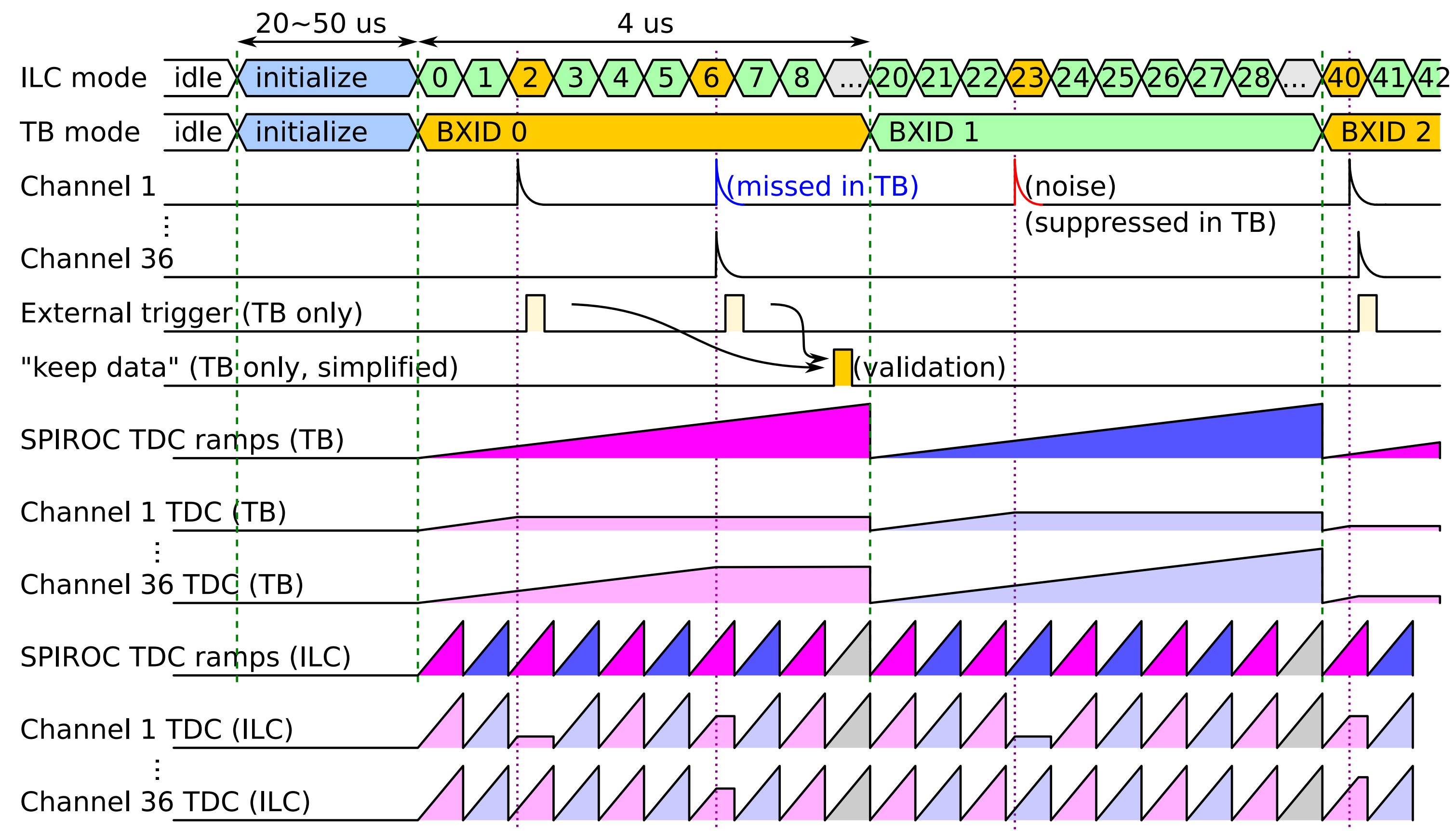




\section{Time-walk effect}

Time-walk effect due to the threshold in the chip

- Low amplitude hits -> slow rise of the signal

- High amplitude hits -> fast rise of the signal

Low amplitude hits -> later trigger
D.S. Carman, CLAS12 CTOF Time-Walk Corrections

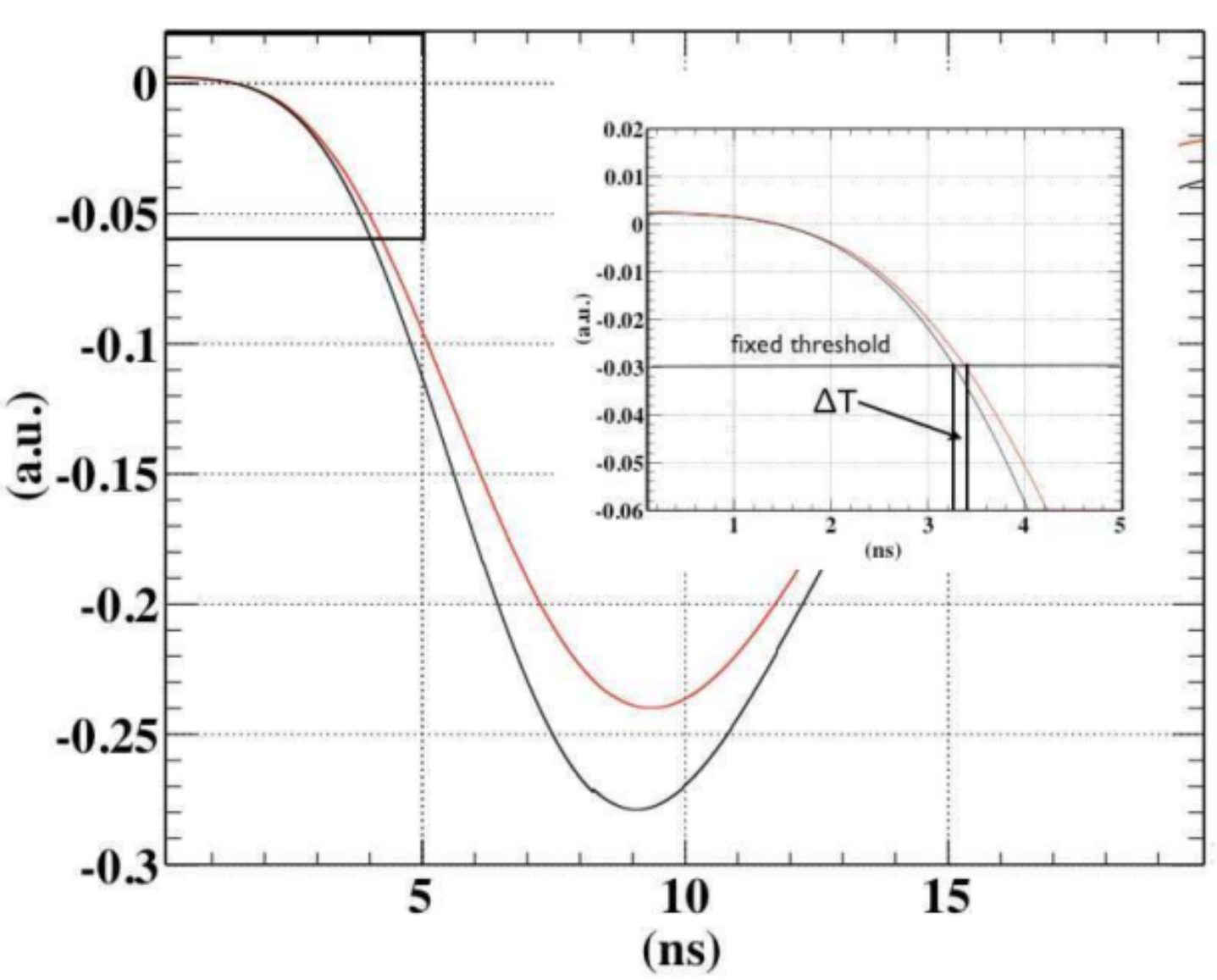




\section{Correction for trigger delay}

An offset is extracted to account for the delay of the trigger

- Trigger logic and cabling

Offset extracted for each chip, channel, memory cell and BXID

Offset per BXID needed!

- Pedestal per memory cells different for each ramp BXID
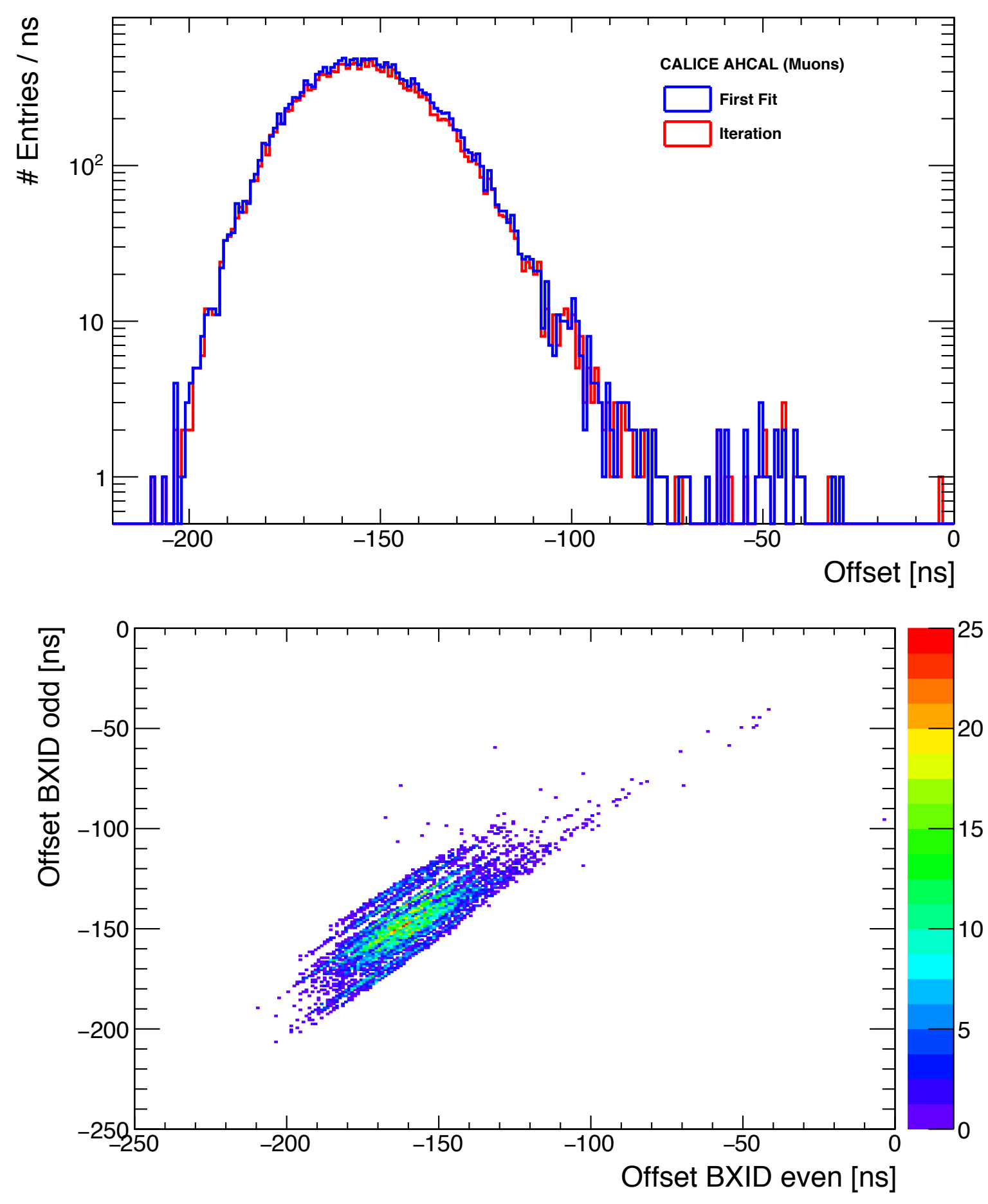


\section{Asymmetry in muons}

Probably due to the non-linearity from the time reference

- Non-corrected for as no external reference

- Dependance visible function of the TDC value of the time reference

- Late hits seems to explain the asymmetry observed $->$ time reference more off along the ramp (lever arm)

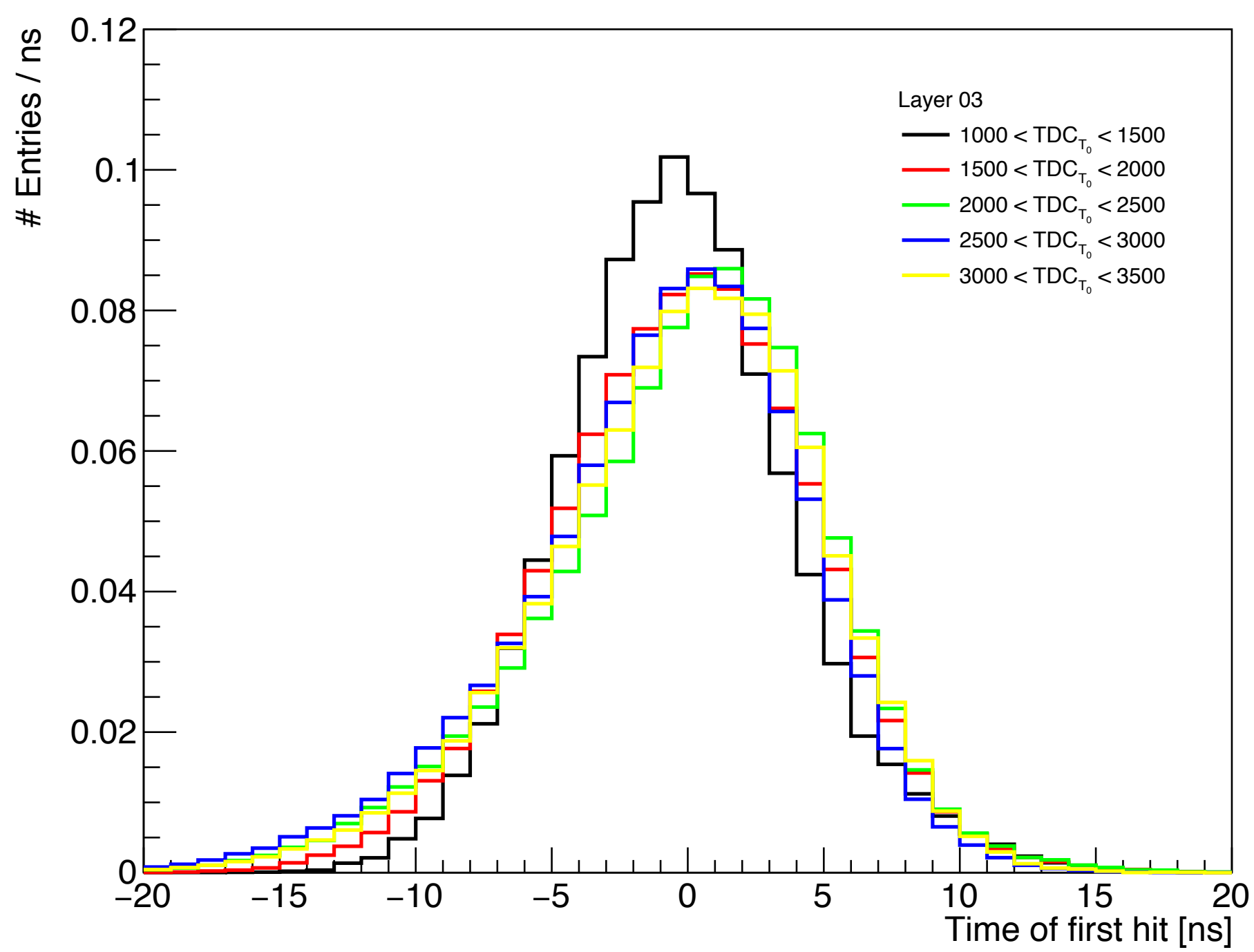




\section{Influence of ROC Digitisation}

Checking influence of the ROC Digitiser on the timing

As expected for muons, no change with threshold

Electrons -> same conclusion
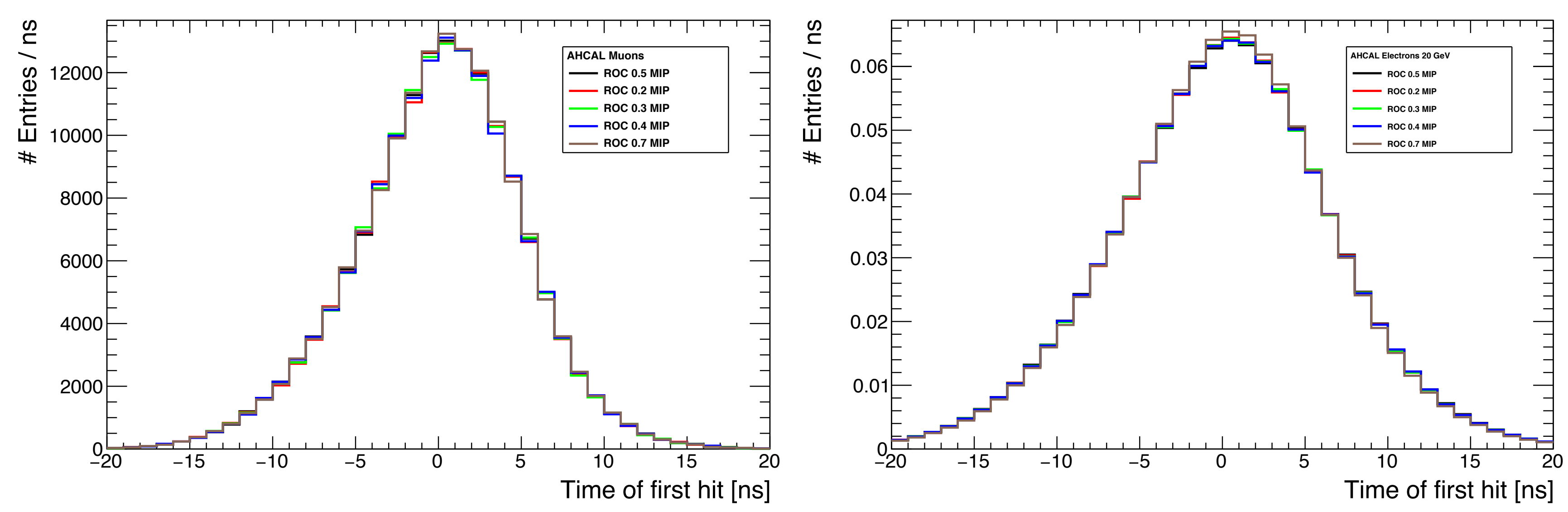